IX.

\title{
Zur Aetiologie und Therapie der progressiven Paralyse.
}

\author{
Von \\ Dr. R. Seeligmann \\ aus Karlstuhe, \\ Assistent an der Heilanstalt "Konstanzer Hof" in Konstanz.
}

Die letzten beiden Decennien und vor allem das letzte Decennium haben eine so grosse Reihe von Arbeiten über das Wesen der progressiven Paralyse, ihren theils bestrittenen, theils behaupteten Zusammenhang mit vorausgegangener Lues gegeben, dass es auf den ersten Blick zum Mindesten unnöthig erscheint, diese überreiche Literatur noch zu vermehren. Wenn dies von meiner Seite dennoch geschieht, so glaube ich dazu berechtigt zu sein, weil zwar die Symptomatologie der Paralyse durch obige Arbeiten genügend beleuchtet erscheint, in der Aetiologie aber und vor Allem in der Therapie dieser Erkrankung eine Reihe strittiger Punkte der Erledigung harren; und weil andererseits das von mir bearbeitete Krankenmaterial in der Eigenart seiner Zusammenstellung von fast nur Frühstadien der progr. Paralyse für die Statistik von ganz besonderem Werth sein dürfte.

Nachdem durch Vorgehen von Erb hauptsächlich durch die Beweiskraft einer auf nnanfechtbare Anamnese gestützten Statistik das Krankheitsbild der Tabes, ihr enger Zusammenhang mit vorausgegangener luetischer Infection festgestellt war, war es begreiflich, dass alsbald auch für das der Tabes verwandte Krankheitsbild der progr. Paralyse die Frage nach der ätiologischen Bedeutung der Lues auftrat. Es ist nicht uninteressant zu beobachten, wie von zunächst fast absoluter Negirung die Ansichten in dieser Frage sich änderten, bis die letzten Jahre uns auch für die Paralyse fast auf den gleichen Standpunkt bringen wie für die Tabes, dass nämlich auch für die progr. Paralyse das weitaus wichtigste prädisponirende Element in der vorausgegangenen luetischen Infection zu suchen ist. Der Grund zu diesem Umschwung liegt zum grossem Theil in dem in den letzten Jahren sorgfältiger ausgesuchten Material der Statistik. Die ersten Statistiken stammen zumeist aus dem Krankenbestande grosser Irrenanstalten, und liegt es auf der Hand, dass ein derartiges an anamnestischen Daten armes Material nur durch Unsicherheit getrübte Ergebnisse liefert; 
daher die kleinen Zahlen ron Eichholtz ${ }^{1}$ ), ca. 12 Proc., Nass e $\left.{ }^{1}\right) 20$ Proc., Reinhard 1) 24 Proc., Goldstein ${ }^{1}$ ) (Privatanstalt) 50 Proc., Ascher ${ }^{2}$ ) (Dalldorf) 40 Proc. u. s. f. Die späteren Autoren, die sich dieser Fehlerquelle bewusst wurden, suchten dieselbe zu vermeiden und benutzten deshalb aus den Irrenanstalten nur die Fälle, über die sichere Daten vorlagen, oder aber die Statistik benutzt frühe Stadien der Erkrankung, wie sie die Praxis der Consiliarien oder die offenen Anstalten bieten, in der die Patienten selbst noch im Stande sind, sichere anamnestische Angaben zu machen. Es ist natürlich, dass solche Statistiken nur über kleinere Zahlen verfügen, aber auch ein kleines, wenn nur sicheres Material wird für die Lues-Paralysefrage nicht zu verachten sein. Spätere Autoren mögen dann durch Zusammenstellung solcher kleinen Statistiken zur Statistik der grossen Zahlen und ihrer beweisenden Kraft kommen.

Die folgende Arbeit, in der in retrospectiver Statistik die Fälle von progr. Paralyse verwerthet sind, die in den Jahren 1877-1897 incl. in den Anstalten von Hofrath Dr. Gg. Fischer, „Wilhelmsbad" in Cannstatt, Heilanstalt "Konstanzer Hof" in Konstanz behandelt wurden, umfasst im ganzen 150 Fälle sicherer progr. Paralyse.

Ehe ich zur Besprechung dieser Fälle übergehe, sei mir noch eine kurze Notiz über die im „Konstanzer Hof" beobachteten Tabesfälle gestattet. Bei der Durchsichtung der Krankengeschichten auf progr. Paralyse mussten auch die Journale der Tabeskranken eingehend behandelt werden, und möchte ich, um das hierbei zu Tage geförderte Resultat für die Statistik nicht verloren gehen zu lassen, dasselbe hier kurz andeuten. Eingeschränkt wird der Werth dieser Zahlen, da bei dem engen Connex der hiesigen Anstalt mit der Consiliar-Praxis verschiedener Universitätsprofessoren - durch Ueberweisung von Fällen - wohl manche Fälle meiner Statistik angehören, die schon anderswo, z. B. in der grossen Statistik Erb's verwerthet sind.

Von 102 Fällen von Tabes, von denen ich zwei - es handelt sich dabei um weibliche Patienten mit unsicheren Angaben - ausschliesse, hatten:

60 ein sicheres Ulcus durum mit secundären) Erscheinungen $=60$ Proc.

18 ein sicheres Ulcus durum, secundäre Erscheinungen unsicher $=18$ Proc.

8 ein Ulcus dubiosum oder Ulcus molle, secundäre

Eirscheinungen fehlten $=8$ Proc.

es waren inficirt 86 Proc.

1) Vgl. Schmidt's Jabrbücher der ges. Medicin. Bd. 210. S. 89. Originalabhandlung von Dr. Rieger.

2) Allgem. Zeitschrift f. Psych. Bd. XIVI. 
14 wurden als frei bezeichnet $=14$ Proc. - doch waren auch hiervon ein Theil durch Placques (3), ein Theil durch Aborte der Frau (2) zum Mindesten verdächtig. -

Indem ich nunmehr zu meinem eigentlichen Thema übergehe, möchte ich zunächst die ätiologischen Fragen eingehend erörtern, um sodann nach kurzer Würdigung der Initialsymptome, in ihrem Werthe für die Frühdiagnose der Paralyse, noch genauer auf die Therapie dieser Erkrankung einzugehen.

Wie ich schon in den einleitenden Worten erwähnte, ist betr. der grossen Wichtigkeit der Lues für die progr. Paralyse eine ziemlich weitgehende Einigung erzielt. Allerdings datirt diese Einigung nur wenige Jahre zurück, denn es sind erst fünf Jahre, seit dem Vorangehen Fournier's ${ }^{1}$ ) und Regis ${ }^{2}$ ) folgend auch die französische Schule ihre Sonderstellung verlassen und sich, sagen wir kurz, der LuesTheorie angeschlossen hat, um dann aber zu den Hauptverfechtern dieser Theorie zu werden und sich der Reihe der extremsten deutschen Forscher anzuschliessen. Hierin deute ich schon an, dass auch unter den deutschen Autoren die Einigung nur eine principielle ist; denn über den Grad des Einflusses der Lues, ob sie das alleinige, oder nur ein häufig mitwirkendes ätiologisches Moment sei, darüber sind die Ansichten noch getheilt. Während Möbius, der auch für die Tabes der Vertreter der unitarischen Lehre geworden, ebenso für die progr. Paralyse das Princip aufstellt, es giebt nur eine progr. Paralyse, die durch Lues bedingte, $d$. $h$. die bei früher luetischen Individuen entstehende, haben eine Reihe anderer Autoren die Lues zwar auch als wichtiges ätiologisches Moment anerkannt, stellen ihr aber eine Reihe theils gleichberechtigter, theils untergeordneter ätiologischer Momente zur Seite; Momente, die besonders in ihrer Combination die Paralyse auch ohne vorausgegangene Lues hervorrufen könnten.

Die Statistik, die berufenste Schiedsrichterin in dieser Frage, liefert noch kein definitives Urtheil, allerdings führen die Resultate der neueren Arbeiten - und auch meine Arbeit dürfte in diesem Sinne verwerthet werden - dem Möbius'schen unitarischen Standpunkte sehr nahe. Die Zahl der lues-freien Patienten ist in genauer Statistik so gering, dass die wenigen Ausnabmefälle fast als beweisend für die Regel angesehen werden dürfen; zumal immer ein Theil dieser Fälle aus verschiedenen, theils beruflichen, theils familiären Gründen und Rücksichten in der wahrheitsgetreuen Angabe anamnestischer Daten zum Mindesten sehr zweifelhaft ist. Dazu kommt noch bei

1) Fournier, Syphỉlis et paralysie générale. Revue neurol. 1893.

2) Regis, Syphilis et paralysie générale. Arch. clin. de Bordeaux 1892 
Paralytikern in nicht ganz frühen Stadien der Erkrankung das erhöhte Selbstbewusstsein der Patienten, die oft die Frage nach einer früheren Infection als beleidigend zurückweisen und jedem weiteren anamnestischen Forschen den hartnäckigsten passiven Widerstand entgegensetzen.

Nicht zum Mindesten aus diesem letzten Punkte, nämlich aus dem möglichsten Vermeiden schon fortgeschrittener Fälle, wie dies in der Eigenart meines Materials bedingt war, erklären sich die relativ hohen Procentziffern Inficirter aus meiner Statistik.

Hier die Ergebnisse:

Von den 150 Fällen, die mir zu Gebote standen, scheide ich $20 \mathrm{Fälle}$, die in der Schwierigkeit der Differentialdiagnose - speciell mit Lues cerebri - das Resultat beeinflussen könnten, aus; in den restirenden 130 Fällen

waren früher sicher luetisch $\quad 95=$ cr. 73 Proc. ) waren inficirt waren früher inficirt (Ulcus dubios.) $10=" 8\}$,81 Proc. waren angeblich frei$$
25=" 19 "
$$

Erhalten wir so schon Zahlen, wie sie den Resultaten von Idanow ${ }^{1}$, Hirschl') und Anderen entsprechen, so steigen meine Zahlen noch, wenn ich, da es sich um retrospective Statistik handelt und in den letzten zehn Jahren noch grösserer Werth auf die Erforschung der Lues gelegt wurde, meine Fälle in die Abschnitte 1878-1887 und 1888-1897 theile.

Die in die Jahre 1878-1887 fallenden 50 Fälle geben natürlich kleinere Procentzahlen, nämlich:

$$
\begin{aligned}
& \text { sichere Lues } 30 \mathrm{mal}=60 \text { Proc. } \\
& \text { Ulcus dubios. } 8,=16 ", \quad \begin{array}{c}
\text { waren inficirt } \\
76 \text { Proc. }
\end{array} \\
& \text { Angeblich frei } 12 "=24
\end{aligned}
$$

dagegen in den den Jahren 1888-1897 angehörigen 80 Fällen

hatten sichere Lues mit nachgewiesenen
sec. Erscheinungen . . . . . $51=63,75$ Proc.

" sicheres Ulcus durum - sec. Er-

$" \quad$ ein Ulcus dubiosum oder Ulcus molle . . . . . . . . $2=2,5$ Proc.

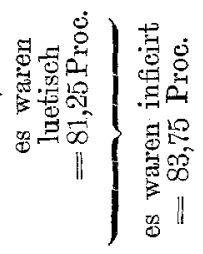

waren frei . . . . . . . $\left.13^{a}\right)=16,25$ Proc.

a) Doch sind hiervon durch Narben, Placques, Aborte der Frau etwa vier Fälle dringend - trotz Negirung der Patienten - der Lues verdächtig.

1) Idanow, De la paralysie générale. Ann, med. psych. XIX. 1894.

2) Hirschl, Die Aetiologie der progr. Paralyse. Jahrbuch der Psych. XIV. 1896. (Material von Krafft-Ebing.) 
Die hiermit gewonnene Procentzahl von fast 84 Proc. Inficirter, die in Anbetracht der dringend luesverdächtigen, unter "frei" aufgeführten Fälle sicher zu niedrig gegriffen und bei Zurechnung nur der verdächtigsten Fälle leicht sich auf 90 Proc. und mehr erhöht, nähert sich dem unitarischen Standpunkt so beträchtlich, dass wir, gestätzt auf unser Material, wohl berechtigt sind, uns ebenfalls auf den Standpunkt einer unitarischen Aetiologie der progressiven Paralyse zu stellen.

Was die so viel betonten ätiologischen Nebenmomente angeht, so wurde auch in unseren Fällen oft genug die bekannte "intellectuelle Ueberanstrengung“, daneben Potus - $18 \mathrm{mal}$ Nicotin - 1 mal - Heredität - 6 mal - beschuldigt. Wenn nun auch kaum zu leugnen ist, dass derartige das gesammte Nervensystem schädigende Einflüsse das Auftreten der Paralyse begünstigen, vielleicht sogar beschleunigen, so erseheint ihre Mitwirkung gegenüber der Hauptätiologie der Lues doch oft überschätzt zu werden. Insbesondere gilt dies für die fast in jeder Anamnese wiederkehrende intellectuelle Anstrengung, die bei genauerer Forschung meist auf ein das Niveau mittlerer geistiger Arbeit nicht übersteigendes Maass herabsinkt; ich kann mich daher in diesem Punkte nur. Dr. Pierson ${ }^{1}$ ) anschliessen, der auch auf die zwar häufige Erwähnung, aber das nur sehr seltene Stichhalten der geistigen Ueberarbeitung hinweist. Von den übrigen Nebenursachen der progr. Paralyse ist wohl am meisten der Alkoholmissbrauch zu beschuldigen, und hier ist die Vermuthung wohl berechtigt, dass dieses Moment häufiger mitwirkt, als es zugestanden wird. Es ist wenigstens zum Mindesten auffallend, dass in all' den allerdings nur wenigen Fällen, in denen Lues negirt wurde, ebenso auch Alkoholmissbrauch, wie überhaupt jeder Missbrauch und Excess geleugnet wird; hier kehrt immer und immer die geistige Ueberanstrengung als alleinige Ursache wieder. Entspricht es doch auch ganz dem erhöhten Selbstbewusstsein des Paralytikers, mit der Schilderung seiner Leistungen zu spielen, sich als Opfer seines Berufes zu betrachten, während er jeden Excess oder Aehnliches als unter seiner Würde liegend betrachtet; ein Grund mehr, den optimistischen Angaben der Pat. gegenüber in jeder Beziehung misstrauisch zu bleiben.

Ehe ich meine ätiologischen Bemerkungen beschliesse, seien mir noch einige Worte zur Frage der Latenzzeit der progressiven Paralyse - der Zeit zwischen luetischer Infection und Ausbruch der Paralyse - gestattet. Diese Latenzzeit schwankt in meinen Beobachtungen zwischen 3 und nahezu 30 Jahren; es scheint, dass sie durch

1) Bericht über die Privatheilanstalt Pirna. - Dresden 1893.

Dentsehe Zeitschr. f. Nervenheilkunde. XIII. Bd. 
anderweitige Schädigung des Nervensystems abgekürzt - und hierin liegt vielleicht die grösste Bedeutung der sog. ätiologischen Nebenmomente - , durch ruhiges, vernünftiges Leben, vor Allem aber durch gründliche Behandlung der primären Infeetion hinausgeschoben werden kann. Jedenfalls verdient es hervorgehoben zu werden, wie die Latenzzeit gerade in den Fällen auffallend kurz war, in denen neben der Lues noch andere Momente wirkten und dazu noch die ursprüngliche Infection nicht oder doch nicht mit $\mathrm{Hg}$ behandelt wurde. Ueberhaupt waren von den 80 Fällen des letzten Decenniums, wobei auf diese Punkte genauer geachtet wurde, $31=$ ca. 40 Proc., deren Infection s.Z. nicht oder doch nicht genügend behandelt worden war. Dies führt uns zu der so wichtigen Frage über die Berechtigung und den Erfolg einer Spätbehandlung der der Paralyse vorausgehenden Lues, einen noch viel umstrittenen Punkt, auf den ich am Schluss meiner Arbeit noch ausführlich zurückkommen werde.

Zunächst einige lrurze Bemerkungen über die Initials ymptome and die klinischen Erscheinungen der besprochenen Fälle.

Seit vor Kurzem Hoche in seiner „Frühdiagnose der progressiven Paralyse" ${ }^{1}$ ) fast alles über dieses Kapitel Bekannte niedergelegt hat, ist es kaum möglich, auf diesem Gebiete noch Neues zu bringen. Trotzdem kann bei der grossen Bedeutung der Frühdiagnose - speciell für den praktischen Arzt - die Wichtigkeit der initialen Symptome nicht oft genug betont, ihre Kenntniss nicht oft genug erweitert werden. Denn noch immer gehören die Fälle nicht zu den Seltenheiten, in denen durch Verkennen paralytischer Symptome die Kranken unter der Diagnose Neurasthenie auf sogenannten Erholungsreisen herumgehetzt werden, bis die Progression des Leidens oder ein günstiger Zufall schliesslich doch die richtige Diagnose stellen lässt. Im günstigsten Falle früh genug, um das Versäumte noch nachholen zu können; oft aber auch erst, nachdem das Verkennen des Leidens dem Kranken schwere körperliche, seiner Familie irreparable sociale Schädigungen beigefügt hat.

Von den Initialsymptomen, die immer und immer wieder in den Vordergrund der Betrachtung gestellt werden müssen, erscheint als wichtigstes psychisches Moment die Charakterveränderung der Erkrankten, sei es im Bilde der reizbaren Schwäche mit ihrer - im Gegensatze zur Neurasthenie - euphorischen Krankheitsverkennung, sei es im Bilde der depressiven Verstimmung mit ihren Selbstanklagen und darin oft bedingten Suicid-Ideen. Diesen meist nur aus den Mittheilungen der engeren Umgebung der Kranken ersichtlichen Aende-

1) Sammlung zwangloser Abhandlungen aus dem Gebiete der Nerven- und Geisteskrankheiten Bd. 1, Heft 1. 1896. 
rungen im Wesen, von denen ich noch die Lenkbarkeit im Grossen, den heftigen, oft zu Scenen führenden Widerstand in Kleinigkeiten hervorheben möchte, steht ein weiteres, wohl noch als psychisch zu bezeichnendes Symptom an Grösse der Bedeutung fast zur Seite, da es in der Anamnese des Patienten selbst sehr häufig, oft als einziges bewusstes und zugestandenes Krankheitssymptom erscheint: die Schlaflosigkeit. Ist eine im besten Mannesalter ohne äussere Gründe auftretende Schlaflosigkeit schon immer verdächtig, so wird sie für progressive Paralyse fast pathognostisch, sobald sie mit bei Tage meist ganz unmotivirt auftretender Schlafsucht verbunden ist. Diese Combination von Schlaflosigkeit bei Nacht mit Schlafsucht bei Tage - die in unseren Fällen in 20 Proc. nachweisbar war - dürfte speciell zur Abgrenzung von Neurasthenie mehr als bisher zu beachten sein.

Die schon zu den somatischen Symptomen überleitenden Initialerscheinungen, wie Kopfschmerz - speciell Migräne - waren bei meinem Material nur 3 mal vertreten, und kann ich bei der Genauigkeit, mit der $\mathrm{Hoche}^{1}$ ) sie behandelt, auf eine eingehende Erörterung an dieser Stelle verzichten.

Unter den eigentlich somatischen Symptomen erscheint, wenn ich zunächst von dem Verhalten der Pupillen und der Reflexe, die später besprochen werden sollen, absehe, als wichtigstes, weil oft folgenschwerstes, der paralytische Anfall - vom einfachen, nur Secunden dauernden Schwindelanfall mit kaum getrübtem Bewusstsein die ganze wechselreiche Stufenleiter hinan bis zum schwersten apoplectiformen Insult. Hieran reihen sich, oft durch diese Anfälle bedingt, in wechselnder Häufigkeit der Erscheinung, Paresen und Parästhesien verschiedener Gebiete, theils passagère, theils dauernde Aphasien und Paraphasien, schliesslich anch Störungen in der Function des Geschlechtsand Harnapparates. Besonders auf dieses zuletzt genannte Symptom möchte ich noch speciell aufmerksam machen; in unseren 80 Fällen erscheint 26 mal Schwäche der Blase als erstes oder doch eines der ersten Symptome erwähnt, oft handelt es sich nur um häufigeres, plötzliches, zeitweise unbewusstes Uriniren, in selteneren Fällen um ausgesprochene Ischuria paradoxa. Ohne diesem Symptom zu grossen Werth beizulegen, so ist doch sein Nachweis bei sonst vorhandenen verdächtigen Momenten für die Diagnose der Paralyse zu verwerthen.

Als ein anderes Symptomenbild des Frühstadiums der Paralyse erwähne ich noch die mit Tabessymptomen beginnende ascendirende Paralyse - Taboparalyse, doch kann ich hier wohl auf eine Schil-

1) l. c. 
derung jener Symptome verzichten, indern ich für den allerersten Beginn der Erkrankung auf das bekannte Krankheitsbild der Tabes, für spätere Stadien und eventuelle Complicationen auf das oben Gesagte verweise.

Der Vollständigkeit wegen sei auch der seltenen Fälle gedacht, in denen die progr. Paralyse sich mit Augenmuskellähmungen Psychosen - oder anderen nervösen und neuralgiformen Symptomen einleitet, doch beschränke ich hier meine Mittheilungen auf zwei Fälle, die allerdings bei der eigenartigen Seltenheit ihrer Erscheinungen verdienen, in extenso wiedergegeben zu werden.

In beiden Fällen, in denen die Diagnose lange schwankte, handelte es sich um eigenartige, zumeist im Abdomen localisirte Beschwerden und Sensationen, die lange Zeit so im Vordergrund standen, dass sie das Bild der Paralyse fast rerdeckten. Hier die diesbezẗglichen klinischen Notizen!

Fall I. Pat. 36 Jahre alt. Lues und Potus negirt; doch sollen früher vorgekommen sein.

Seit zwei Jahren Beschwerden im Anschluss an geistige Ueberanstrengung. Vor 1/2 Jahr Sturz vom Pferde, angeblich in Folge plötzlichen Schwindels beim Reiten.

Aufnahme bei Herrn Hofrath Eischer.

Klagen über zeitweisen Schwindel, vor Allem aber über Besehwerden im Abdomen. Unregelmässigkeit in der Verdauung. Zeitweise - alle 5-6 Tage - krampfartige Schmerzen im Abdomen, Milzgegend, dabei Frostgefühl. - Cyanose des Gesichts.

Diese Anfälle beherrschen das Krankheitsbild.

Status.

Von Seiten der inneren Organe nichtNennenswerthes. Gehirnnerven intact.

Pupillen etwas eng; reagiren, wenn auch langsam, Augenbewegungen prompt. Kein Nystagmus.

Sensibilität - Schmerzleitung u. s. f. normal.

Reflexe: Arm- und Patellarreflexe $r .=1$, lebhaft.

Psyche - etwas depressive Verstimmung, keine charakteristische Veränderung quoad Paralyse.

Damalige Diagnose: Neurasthenie. Betheiligung des sympathischen Systems - N. Splanchnici.

Verdacht auf Paralyse.

Nach zweimonatlichem Aufenthalt etwas gebessert entlassen, traten bald die alten Beschwerden verstärkt wieder auf, auch stehen noch immer im Vordergrund die krampfartigen Sensationen im Abdomen; daneben zeitweise Kopfschmerz, Stockungen in der Sprache und jetzt - nach etwa $1 / 2 \mathrm{Jahr}$ - auch deatliche psychische Veränderungen. Pat. wurde leichter reizbar, in seinen Stimmungen schwankend, nach und nach Abnahme der geistigen Functionen und nach etwa einem Jahr dentlich ausgesprochene progr. Paralyse, die wegen maniakalischer Erscheinungen die Aufnahme in der Irrenanstalt nöthig machte. 
Die Notizen über den Verlanf ans der Anstaltszeit, die mir durch die Freundlichkeit von Herrn Professor Binswanger zugänglich waren, zeigen das bekannte wechselreiche Krankheitsbild der Paralytiker.

Zuerst Erregungszustände verschiedener Art mit Hallucinationen, die mit ruhigeren Epochen abwechseln, schliesslich mehr und mehr schwinden, um von totaler Verblödung gefolgt zu werden.

Betr. der körperlichen Symptome wäre nur zu bemerken, dass die erwähnten Sensationen allmählich mit dem Ueberhandnehmen der psychischen Symptome verschwanden; sonst war Aussergewöhnliches nicht zu bemerken.

Nach ca. 4jährigem Leiden - die beiden letzten Jahre in der Irrenanstalt - trat der Exitns ein.

Section wurde verweigert.

Fall II. Pat. 40 Jahre alt. Lues vor 20 Jahren - mit $\mathrm{Hg}$ behandelt. Potus.

Seit vier Jahren Beschwerden von Seiten des Abdomens. Pat. klagte zunächst über eigenartige, schwer zu definirende, quälende Sensationen im Abdomen, besonders in der linken Bauchseite; dazu trat in den letzten Monaten, angeblich durch diese störenden Gefühle bedingt, Schlaflosigkeit.

Aus diesen Gründen suchte Pat. unsere Anstalt auf; die genannten Beschwerden blieben vorherrschend, ohne dass die körperliche Untersuchung eine Erklärung gegeben. Der Status der inneren Organe, ebenso das Nervensystem erschien normal, nur war die r. Pupille etwas $>1$, ihre Reaction etwas träger als normal.

Das psychische Verhalten des Pat. war das eines nervösen, leicht erregten Mannes, gab aber keinerlei Charakteristica für progr. Paralyse.

Die Diagnose blieb zunächst unsicher; die Schmerzen im Abdomen liessen an Visceral-Neuralgien denken, das übrige Wesen des Pat. war das eines Neurasthenikers; allerdings war die hartnäckige Schlaflosigkeit auffallend und für Paralyse verdächtig.

Díe spätere Entwicklung bestätigte diesen Verdacht, schon nach wenigen Monaten trat die in der Anstalt etwas gebesserte Schlaflosigkeit in verstärktem Masse auf, es gesellte sich bald unmotivirte bei Tage anftretende Schlafsucht hinzu. Etwa 1/4 Jahr später kurzer Schwindelanfall mit passagèrer Sprachstörung, und im Anschluss daran rascher Fortschritt der Beschwerden, bis in etwa 1-2 Jahren sich das Krankbeitsbild dem der Paralyse mehr und mehr näherte.

Drei Jahre nach der ersten Aufnahme tritt Pat. wieder in unsere Anstalt ein.

Die früher im Vordergrund gestandenen Klagen über die Sensationen im Abdomen treten mehr zurück. Die einzigen Klagen des Pat. beziehen sich auf seine Schlaflosigkeit und die zeitweise auftretende Unsicherheit der Sprache. Auffallend verändert ist das Wesen des Pat., er gefällt sich im Posiren, in allerlei stereotypen Redensarten u. s. f., kurz der erste Eindruck ist, dass man es mit einem ausgesprochenen Paralytiker zu thun hat. Das weitschweifige Erzählen, das Silbenstolpern und nicht zum Mindesten auch die somatischen Symptome - Pupillenstarre - Steigerung der Reflexe - Parese der Blase u. A. - bestätigten die Diagnose. Ebenso der weitere Verlauf; nach weiteren zwei Jahren traten Tobsuchts- 
anfälle auf, die die Verbringung in eine geschlossene Anstalt nöthig machten; hier trat nach fast 3jährigem Aufenthalt im Stadium vollkommener Verblödung der Tod ein.

In beiden vorliegenden Fällen handelt es sich, um dies nochmals hervorzuheben, um sichere typische progr. Paralyse, nur mit eigenartigen Einleitungssymptomen, den visceralen Sensationen; ob diesen eine grössere Bedeutung beizumessen ist, ob sie den mit Magenkrisen beginnenden Paralysen zur Seite zu stellen oder mehr als psychisch bedingt anzuseben, wage ich nicht zu entscheiden, und wollte ich diese Fälle ihrer Eigenart wegen nur erwähnen.

Was den ziemlich protrahirten. Verlauf des Falles II betrifft gehen wir bis zum ersten Auftreten der Sensationen zurück, so waren es zehn Jahre -, so führt mich dies zur Frage nach der Dauer der Paralyse überhaupt, nach dem oft Jahre lang der ausgesprochenen Erkrankung vorausgehenden Auftreten einzelner Symptome oder Symptomencomplexe. Von Thomsen ${ }^{1}$ ) wurde vor Kurzem darauf aufmerksam gemacht, wie viele Jahre - bis zu 30 - einzelne Erscheinungen dem dentlichen Ausbruch der Erkrankung vorausgehen können; wenn mein Material so grosse Intervalle auch nicht giebt, so finden sich doch darin eine Reihe von Beobachtungen, in denen Schwindelanfälle, Apoplexien mit Sprachstörungen Jahre lang - bis zu 10 Jahren - als einzige verdächtige Symptome bestanden; ebenso ging einmal eine mit Pupillenstarre verbundene Diplopie lange Zeit dem Ausbruch der Paralyse voraus. Diese Fälle müssen erwähnt werden, um der speciell bei vielen praktischen Aerzten, aber auch in Besprechungen dex Paralyse noch herrschenden Ansicht entgegenzutreten, die Daner der Paralyse beschränke sich auf 2-3 Jahre. Mit dieser Ansicht, die mit der Diagnose „Paralyse" dem Kranken für kurze Zeit das Todesurtheil fällt, sind schon viele sociale Missstände geschaffen worden; es kann deshalb nicht genug betont werden, dass die progr. Paralyse keineswegs eine Krankheit sehr kurzer Dauer ist, protrahirte Fälle von 6-10 Jahren gehören keineswegs zu den Seltenheiten, ja es sind Fälle bekannt, die noch längere Zeit andauerten.

Dem oben über die Symptomatologie Gesagten möchte ich nur noch einige statistische Bemerkungen über das Verhalten der Reflexe, das Verhalten der Pupillen anfügen; alle übrigen Symptome der Paralyse, wie das Silbenstolpern, die eigene Mimik beim Sprechen, die Paresen der verschiedenen Hirnnerven und Anderes darf ich wohl als bekannt voraussetzen und darf deshalb auf eine diesbezügliche Besprechung verzichten.

1) Thomsen, Allg. Zeitsch. für Psych. Bd. LII. 1896. 
Es fand sich in 80 Fällen

Reflectorische Starre oder Trägheit der Pupillen . . . . 57 mal

54 mal beider Augen, 3 mal eines Anges.

Normale Reaction . . . . . . . . . . . . . . . $18 \mathrm{mal}$

Angaben unsicher oder nicht prüfbar . . . . . . . . 5 mal

dabei:

Pupillendifferenz . . . . . . . . . . . . . . . $50 \mathrm{mal}$

Augenmuskellähmongen . . . . . . . . . . . . . 5 mal

Nystagmus . . . . . . . . . . . . . . . . . 2 mal.

In den gleichen Fällen waren die

Patellarreflexe:

normal $14 \mathrm{mal}$

gesteigert $44 \mathrm{mal}$

Armreflexe:

normal $10 \mathrm{mal}$

40 mal beiderseits,

darunter 3 mal klonisch, 4 mal einseitig.

herabgesetzt $5 \mathrm{mal}$ herabgesetzt $5 \mathrm{mal}$ fehlend 16 mal fehlend $12 \mathrm{mal}$ nicht zu prüfen 1 mal. nicht zu prüfen 10 mal.

Das Verhalten der Pupillen und Reflexe gleichzeitig ergab folgende Combination.

Bei reflectorischer Starre waren die Patellarreflexe

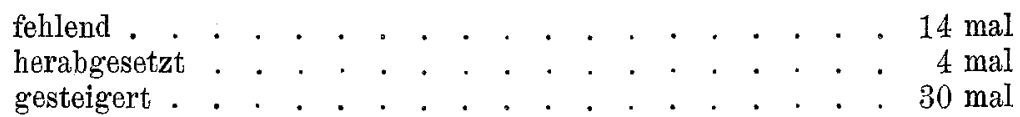

bei normaler Pupillenreaction waren die Patellarreflexe

fehlend . . . . . . . . . . . . . . . . . . . 4 mal gesteigert . . . . . . . . . . . . . . $10 \mathrm{mal}$ normal . . . . . . . . . . . . . . . . . . . 4 mal

d. h. normales Verhalten der Pupillen und Reflexe in nur $4=5$ Proc. aller Fälle.

Die obigen Zahlen ergeben das gewohnte und bekannte Resultat, das ausser für Tabes auch für progr. Paralyse charakteristische Vorkommen reflectorischer Pupillenstarre, das, mit Feblen der Patellarreflexe verbunden, für diese Krankheiten geradezu pathognostisch ist. Weniger wichtig, da zu sehr der subjectiven Beurtheilung des einzelnen Untersuchers anheimgegeben, ist die Verbindung reflectorischer Starre mit gesteigerten Reflexen; immerhin darf, bei dem seltenen Fehlen beider Symptome, auch diesem Symptomenconnex insofern Bedeutung beigemessen werden, als er den Verdacht auf progr. Paralyse erwecken muss und deshalb auch für spätere Zeiträume zu weiterer genauer Beobachtung anregen muss. Für eine Eintheilung in gewisse Stadien der Paralyse liess sich in unseren Fällen weder das Verhalten 
der Pupillen - etwa im Sinne von Bevan Lewis $\left.{ }^{1}\right)$, Oliver ${ }^{2}$ ) u. a. Aut. - noch das Verhalten der Reflexe verwerthen.

Hiermit schliesse ich meine symptomatologischen Notizen, die, möglichster Kürze wegen, nur einiges bisher weniger Bekanntes prägnanter hervorheben sollten, und gehe zu dem letzten Theile meiner Arbeit über, zur Frage nach der Therapie der Paralyse.

Seit der Zusammenhang zwischen Lues und progr. Paralyse durch die Statistik mehr und mehr wahrscheinlich wurde, lenkte sich auch das Auge des Therapeuten mehr dieser Frage zu, und es entwickelte sich hieraus die prophylaktische und causale Behandlung der Paralyse. Wenn auch für den Werth der Prophylaxe beweisende Zahlenreihen nicht anzuführen sind, so scheint es doch auffallend, welche grosse Zahl ron Paralytikern in ihrer Anamnese theils mangelhaft, theils überhaupt nicht behandelte Lues aufweisen. Wir dürfen deshalb, zumal bei der geringen Bedeutung sonstiger ätiologischer Momente, bei der Schwierigkeit, für diese eine Prophylaxe zu üben, auch für die progr. Paralyse den gleichen Satz aufstellen, den Erb ${ }^{3}$ ) in seinem Vortrag über Tabes niederlegte, dass nämlich die einzig richtige Prophylaxe in einer lange fortgesetzten und gründlichen Behandlung der Frühstadien der Lues besteht.

Dass man daneben versuchen wird, durch Fernhalten anderer schwächender Einflüsse das Nervensystem derart disponirter Patienten möglichst kräftig zu erhalten, ist wohl selbstverständlich, doch nur zu oft dürfte der Einfluss des Arztes auf die Lebensweise des Pat. nicht genügend sein, um das erstrebte Resultat zu erreichen.

Ist die Zeit der Prophylaxe unbenutzt vorübergegangen, und haben wir mit der ausgebrochenen Erkrankung zu rechnen, so tritt die causale, d.h. die specifische Behandlung in ihre Rechte. Die antisyphilitische Behandlung der Paralyse war nach erfolgtem Nachweis des Zusammenhangs der Lues und der Paralyse seiner Zeit mit grossen Hoffnungen begonnen worden, leider waren die damaligen Erfolge keineswegs ermuthigend, und die Behandlung wurde ebenso rasch, wie sie gekommen, wieder verlassen, um selbst bis in die neueste Zeit ron so gewichtigen Autoren, wie Krafft-Ebing und Möbius, als direct schädlich bekämpft zu werden. Erst in der neuesten Zeit mehren sich die Veröffentlichungen, die von Neuem auf den Werth der specifischen Behandlung aufmerksam machen, und der Hauptzweck dieser Arbeit soll zunächst

1) Bevan Lewis, Brit. med. Journ. 25. April 1896.

2) Oliver, Med. News. 11. - 1893.

3) Erb, Die Therapie der Tabes. Deutsche Zeitschr. für Nervenheilkunde. Bd. XI. 
sein, zu erneuten Versuchen mit der antisyphilitischen Behandlung der progr. Paralyse anzuregen. Allerdings ist es schwer, bei einem so wechselreichen, an und für sich schon zu Remissionen neigenden Krankheitsbild Beweise für die Erfolge einer Therapie zu bringen, eine Reibe ron Beobachtungen aus meinem Material zwingen jedoch zu der Annahme, dass die durch Hg-Curen erzielten Remissionen als aussergew öhnliche anzusehen sind. Es scheint darnach ein günstiger Einfluss der $\mathrm{Hg}$-Cur für gewisse Fälle kaum zu leugnen, zum Mindesten ist aber - eine verntüftige Auswahl der Patienten vorausgesetzt - eine Schädigung bei der specifischen Therapie mit Sicherheit auszuschliessen. Zweifelhaft muss dagegen die Frage bleiben, ob wirkliche Heilungen der progr. Paralyse, wie solche in letzter Zeit mehrfach berichtet werden, durch $\mathrm{Hg}$ und $\mathrm{JK}$ erzielt werden können; hier erscheint jedenfalls ein gewisser Skepticismus am Platze. So leicht die Diagnose der Paralyse, wenn der Verlauf in der Progression des Leidens die Diagnose bestätigt, so schwer ist sie, falls dieses Moment fehlt; hier sind die differentialdiagnostischen Schwierigkeiten oft unüberwindlich, und mancher Fall von geheilter Paralyse wird in dieser Unsicherheit der Diagnose seine Erklärung finden.

Unter meinem Material fanden sich 3 solcher Fälle, die durch psychische und somatische Symptome den Verdacht auf Paralyse, ja die Diagnose der Paralyse fast aufzwangen; auf eine gründliche $\mathrm{Hg}$ Behandlung schwanden alle oder doch fast alle Symptome, und alle 3 Patienten leben noch jetzt und sind geachtete Glieder der Gesellschaft. - In Fall III ist eine Remission nicht ganz ausgeschlossen, da erst 11/2 Jahre seit der Entlassung verstrichen sind. - Ob nun diese Fälle als Heilungen von progr. Paralyse oder aber als dieser nur ähnliche, durch Lues cerebri bedingte Krankheitserscheinungen aufzufassen sind, wage ich nicht $\mathrm{zu}$ entscheiden. Ich lasse die betreffenden Krankengeschichten folgen, da diese diagnostischen Bedenken ebenso wie der durch $\mathrm{Hg}$ beeinflusste Verlauf allgemeines Interesse verdienen.

Fall I. Pat. 34 Jahre alt. Vor acht Jahren Ulcus durum, später Halsaffectionen. Hautausschläge. Kurze Hg-Cur, ca. 20 Einreibungen, dann kurze Zeit Injectionen. Hereditär schwer belastet. Sexuelle Excesse? Kein Potus.

Seit Monaten Klagen über Schlaflosigkeit, leichte Ermüdbarkeit. Verändertes, zum Jähzorn neigendes Wesen, Angstgefühl, zeitweise depressive Verstimmung.

Seit kurzer Zeit Abnahme der Potenz.

Vor wenigen Wochen Schwindelanfall. Ob mit Sprachstörung, ist nicht ganz sicher zu ermitteln.

Status.

Innere Organe ohne Besonderheiten. 
Pupillen 1.>r., reagiren träge auf Licht und Beschattung, prompt auf Accomodation.

Sonstige Hirnnerven, besonders Facialis und Hypoglossus, frei.

Reflexe an den Armen fehlend; Patellarreflexe lebhaft gesteigert.

Sensibilität intact - Schmerzleitung an den Beinen etwas herab. gesetzt.

Sprache suspect - bei schwierigen Proben.

Rechnen unsicher.

Psyche auffallend weinerlich verstimmt. Leichter Intelligenzdefect.

Nach etwa sechs Wochen heftiger Schwindelanfall, dabei etwas getrübtes Bewusstsein; nach dem Anfall geringe Parese des r. Facialis.

Intensive Hg-Schmiercur $(250 \mathrm{~g})$, dann längere Zeit JK.

Langsames Zurückgehen der Beschwerden, Schlaf tritt wieder ein, die Sprache wird freier und die Stimmung bessert sich etwas. Leichtere Schwindelanfälle mit zeitweisen Rï̈ckfällen wiederholen sich öfter.

Pat. stellt sich im folgenden Jahr wieder vor, ist entschieden in seinem Wesen freier, kann wieder arbeiten; seine Klagen beschränken sich auf ein "unbehagliches Schwindelgefühl, das ihn nie ganz verlässt". Trotz der deutlichen Besserung oder wegen derselben abermalige Hg-Cur, gefolgt von J.K-Cur.

Keine Aenderungen im Status.

Wiedervorgestellt nach zwei Jahren, macht den Eindruck eines gesunden Mannes, nur zeitweise in seinem Benehmen etwas unsicher, sonst Psyche intact.

Sprache ganz frei.

Pupillendifferenz nach wie vor, ebenso die träge Reaction.

Keine Facialisparese mehr.

Reflexe nicht mehr gesteigert.

Nach weiteren zwei Jahren tritt Pat. zu nochmaliger Beobachtung wieder in die Anstalt ein, seine Klagen: Ohrensausen, unruhiger Schlaf $\boldsymbol{u}$. a. sind die Folge grösserer Arbeiten der vergangenen Jahre.

Keinerlei psychische oder somatische Symptome mehr, die auf Paralyse hinweisen.

Pupillendifferenz nicht mehr deutlich nachweisbar; Reaction nur bei schlechter Belenchtung noch träge. Sonst nichts. Der gleiche negative Befund wird bei einer späteren Untersuchung, im vorigen Jahre erhoben.

Fall II. Pat. 33 Jahre alt. Vor zehn Jahren Ulcus durum mit secundären Erscheinungen. Kurze $\mathrm{Hg}$-Cur. Excesse in potn zugestanden. Keine Belastung.

Seit ca. zwei Jahren im Anschluss an grössere körperliche und geistige Anstrengungen - hat sich künstlich durch Wein und Kaffee-Genuss aufrecht gehalten - Veränderung im Wesen des Pat, er wurde apathisch: indolent gegen die Familie.

Vor $1 / 2$ Jahre plötalicher Schwindelanfall beim Aufstehen, Bewusstsein war für einige Zeit verloren; später trat heftiges Erbrechen auf. Nach einigen Tagen getrübten Sensoriums fand sich eine deutliche Sprachstörung, Parese im 1. Facialis und geringe Schwäche im l. Arm, ferner Abducensparese rechts.

Auf eine gründliche Hg-Behandlung schwanden fast alle körperlichen Symptome, dagegen blieb das psychische Verhalten des Pat. verändert. 
Der etwa $1 / 2$ Jahr nach dem Insult im Konstanzer Hof aufgenommene Status ergab fast keine objectiven Veränderungen.

Status.

Innere Organe normal.

Pupillen gleich - Reaction prompt bei starker plötzlicher Beleuchtung, bei Tageslicht träge, bei Beschattung fast nicht nachweisbar (rechts besser als links).

Facialis links gering paretisch.

Armreflexe deutlich, Patellarrefiexe wechselnd, zeitweise mit Jendrassik fehlend, dann wieder auszulösen, jedenfalls stark herabgesetzt.

Sprache frei.

Psyche auffallend; Pat. benimmt sich nicht seiner Stellung angemessen, ist aufdringlich, besonders dem weiblichen Geschlecht gegenüber, gefällt sich in weitschweiffen, stereotypen Redensarten, kurz macht ganz den Eindruck des Paralytiliers.

Bei dem Erfolg der ersten Hg.Cur wurde eine solche hier wiederholt und später JK gegeben.

Der Erfolg blieb zunächst aus, die somatischen geringen Beschwerden, ebenso das auffallende psychische Verhalten blieb.

Später trat dann jedoch eine ganz auffallende Besserung ein, der Pat. konnte seinen beruflichen Pflichten wieder nachkommen und ist, wie ich brieflichen Mittheilungen entnehme, auch jetzt nach fast sechs Jahren noch als vollkommen gesund zu bezeichnen.

Fall III. Pat. 40 Jahre alt. Vor 20 Jahren Uleus durum mit geringgradigen Secundärerscheinungen. Mangelhaft behandelt. Excesse in venere. Kein Potus. Geringe hereditäre Belastung.

Vor etwa einem Jahre, angeblich im Anschluss an grosse Arbeiten, mehrfach leichte Schwindelanfälle. Dann in kurzen Zwischenräumen drei grössere Anfälle mit Bewusstseinstrübung und meist kurzen Sprachstörungen. Vor 1/2 Jahr schwerer Anfall, Sensorium einige Tage benommen. Unsicherheit in Gehen. Aphasie. Gleichzeitig entstand etwa seit einem Jahre eine Veränderung in der Natur des Pat. Reizbarkeit and unmotivirte Depressionen wechselten in rascher Folge. Die Diagnose ist unsicher, neigt nach Ansicht verschiedener Aerzte jedoch zur Paralyse in frühem Stadium. Pat. tritt in unsere Anstalt ein, um hier eine ausgiebige $\mathrm{Hg}$-Cur zu machen.

Der damalige Status ergab:

Auffallend erregtes Wesen, leichter Widerspruch. Beim Erzählen sofort starke articulatorische Sprachstörung, die in der Hast der Schilderung seiner Krankheit sich noch steigert. In seiner Erzählung weitsehweifig; leicht abzulenken, dann wieder unmotivirt heftig werdend. Von körperlichen Symptomen:

Differenz der Pupillen 1.>r. Reaction träge auf Licht, prompt auf Accomodation.

Rechter Facialis etwas paretisch.

Zunge weicht nach rechts $a b$, deutlich fibrilläres Zittern.

Die ïbrigen Hirnnerven intact.

Armreflexe deutlich; Patellarreflexe lebhaft.

Sonst nichts Abnormes.

Unter der Hg-Cur langsame Besserung, die während der späteren JK-Behandlung fortschreitet. 
Am längsten besteht die Paraphasie, doch auch diese gleicht sich ebenso wie die Veränderung im Naturel des Pat. allmählich aus.

Zeitweise während der Car kurze Rückfälle, im Anschluss an Schwindel; ein solcher Anfall war von einer einige Zeit bestehenden Blasenschwäche gefolgt, doch auch diese ging wieder zurück.

Bei einer erneuten Status-Aufnahme nach etwa einem Jahrewar Pat. psychisch ganz intact, anch die Sprache war fast frei, es bestand nur noch eine leichte Ermüdbarkeit and zeitweise Schlaflosigkeit.

Objectiv waren die Symptome nahezn stationär geblieben, nur die Reaction der Pupillen schien prompter geworden.

Abermalige $\mathrm{Hg}$-Cur, von weiterem JK-Gebrauch gefolgt.

Keine wesentlichen Aenderungen im psychischen und somatischen Verhalten.

Nach der Entlassung befindet sich Pat. wohl und ist jetzt seit einigen Monaten ohne Störung in seinem Berufe thätig.

Ich habe diese Krankengeschichten in extenso wiedergegeben, weil sie am besten den eclatanten Erfolg der specifischen Behand$\mathrm{lung}$ in einem der progr. Paralyse zum Mindesten nahe stehenden Symptomencomplex beweisen, weil sie uns zeigen, wie auch nur der Verdacht auf Paralyse in ihren Frühstadien zu einer gründlichen antisyphilitischen Behandlung anregen muss.

Fasse ich die Ergebnisse meiner Beobachtungen, dje Resultate anderer Veröffentlichungen über die Erfolge der specifischen Behandlung der progr. Paralyse kurz zusammen, so lassen sich die Indicationen dieser Behandlung am besten in einigen Sätzen zusammenstellen, am prägnantesten wohl in der Form, wie Erb1) die Indicationen der antisyphilitischen Behandlung der Tabes präcisirt hat.

Ich möchte den heutigen Stand dieser Frage etwa so fixiren:

Bei progressiver Paralyse ist im Allgemeinen die antisyphilitische Behandlung bei vorausgegangener Infection angezeigt, natürlich unter peinlicher Auswahl der einzelnen Fälle.

Speciell eignen sich dazu: alle Frühstadien der Paralyse; hierzu rechne ich auch die verdächtigen Initialstadien mit unsicherer Diagnose, bei denen der Zeitpunkt der Infection nicht zu weit zurückliegt.

Ferner ganz besonders die Fälle, in denen seiner Zeit die luetische Infection nicht oder nur mangelbaft behandelt wurde.

Nochmals sei hervorgehoben, dass eine Schädigung der Kranken durch eine specifische Cur - genügende Individualisirung der einzelnen Fälle - peinliche Ueberwachung während der Hg-Cur vorausgesetzt - fast absolut auszuschliessen ist; jedenfalls ist diese Gefahr eine

1) Erb, 1, c 
minimale in Anbetracht der Erfolge der antisyphilitischen Behandlung gegenüber der aussichtslosen sonstigen Therapie der Paralyse. Beschränkt diese sich doch zum grossen Theil nur darauf, von dem Pat. die Schädigungen fernzuhalten, die die Veränderung seines Charakters - Lenkbarkeit in Vermögensfragen, Grössenideen u. s. f. - in socialer Hinsicht mit sich bringt, ihn fern von diesen Gefahren, fern von seinem Beruf und seiner Familie zu beschäftigen. Wie wenig quoad restitutionem, wie viel im Sinne guter Pflege und Ueberwachung hier die Anstaltsbehandlung. leistet, ist bekannt. Gelingt es doch nicht selten durch genügende leichte Beschäftigung, durch einen festen Tagesplan selbst in der offenen Anstalt den Paralytiker für einige Zeit noch zur Ordnung anzuhalten. Leider ist dieser Erfolg meist nur von kurzer Dauer, bald tritt der progressive Charakter des Leidens von Neuem in seine Rechte, und damit beginnt für den Paralytiker die letzte Stufe seines Leidens: das langsame Hinsiechen in der Irrenanstalt, sei es im Stadium maniakalischer Erregung, sei es im Zustande totaler Verblödung. 